\title{
COST-EFFICIENT RESIDENTIAL ENERGY MANAGEMENT SCHEME FOR INFORMATION-CENTRIC NETWORKING BASED HOME NETWORK IN SMART GRID
}

\author{
Keping $\mathrm{Yu}^{1}$, Battulga Davaasambuu ${ }^{2}$, Nam Hoai Nguyenand ${ }^{2}$, Quang Nguyen ${ }^{2}$, \\ Arifuzzaman Mohammad ${ }^{3}$ and Takuro Sato ${ }^{1,2}$ \\ ${ }^{1}$ Graduate School of Global Information and Telecommunication Studies, Waseda \\ University, Tokyo, Japan \\ ${ }^{2}$ Global Information and Telecommunication Institute, Waseda University, Tokyo, Japan \\ ${ }^{3}$ Faculty of Engineering \& Applied Science, Memorial University of Newfoundland, \\ St. Jon's, Canada
}

\begin{abstract}
Home network (HOMENET) performs multiple important functions such as energy management, multimedia sharing, lighting and climate control in smart grid (SG). In HOMENET there are numerous challenges among which mobility and security are the basic requirements that need to be addressed with priority. The information-centric networking (ICN) is regarded as the future Internet that subscribes data in a content-centric manner irrespective of its location. Furthermore, it has pecial merit in mobility and security since ICN supports in-network caching and self-contained security, these make ICN a potential solution for home communication fabric. This paper aims to apply the ICN approach on HOMENET system, which we called ICN-HOMENET. Then, a proof-of-concept evaluation is employed to evaluate the effectiveness of the proposed ICN-HOMENET approach in data security, device mobility and efficient content distribution for developing HOMENET system in SG. In addition, we proposed a cost-efficient residential energy management (REM) scheme called ICN-REM scheme for ICN-HOMENET system which encourages consumers to shift the start time of appliances from peak hours to off-peak hours to reduce the energy bills. To the best of our knowledge, this is the first attempt to propose an ICN-based REM scheme for HOMENET system. In this proposal, we not only consider the conflicting requests from appliances and domestic power generation, but also think the energy management unit (EMU) should cooperate with measurement sensors to control some specific appliances in some specific conditions. Moreover, the corresponding performance evaluation validates its correctness and effectiveness.
\end{abstract}

\section{KEYWORDS}

Residential Energy Management, Information-Centric Networking, Home Network, Smart Grid

\section{INTRODUCTION}

The worldwide energy consumption is fast increasing. It results in the existing power grid is very difficult to generate necessary enough energy by base plants to address the power demands, and keep the generated power supply and the load demand balanced. Hence, several major blackouts have been already experienced worldwide. Moreover, the existing power grid is almost being used for a century and it shows signs of aging, it is highly required to be innovated right now. 
International Journal of Computer Networks \& Communications (IJCNC) Vol.8, No.2, March 2016

Table1. Comparison of Today's Grid vs. Smart Grid [1]

\begin{tabular}{|c|c|c|}
\hline Preferred Characteristic & Today's Grid & Smart Grid \\
\hline $\begin{array}{l}\text { Active Consumer } \\
\text { Participation }\end{array}$ & $\begin{array}{l}\text { Consumers are uninformed } \\
\text { and do not participate }\end{array}$ & $\begin{array}{c}\text { Informed, involved } \\
\text { consumers-demand response } \\
\text { and distributed energy } \\
\text { resources }\end{array}$ \\
\hline $\begin{array}{l}\text { Accommodation of all } \\
\text { generation and storage } \\
\text { options }\end{array}$ & $\begin{array}{l}\text { Dominated by central } \\
\text { generation-many obstacles } \\
\text { exist for distributed energy } \\
\text { resources interconnection }\end{array}$ & $\begin{array}{l}\text { Many distributed energy } \\
\text { resources with plug-and-play } \\
\text { convenience focus on } \\
\text { renewables }\end{array}$ \\
\hline $\begin{array}{l}\text { New products, services, and } \\
\text { markets }\end{array}$ & $\begin{array}{l}\text { Limited, poorly integrated } \\
\text { wholesale markets. Limited } \\
\text { opportunities for consumers }\end{array}$ & $\begin{array}{l}\text { Mature, well-integrated } \\
\text { wholesale markets. Growth of } \\
\text { new electricity markets for } \\
\text { consumers }\end{array}$ \\
\hline $\begin{array}{l}\text { Provision of power quality for } \\
\text { the digital economy }\end{array}$ & $\begin{array}{l}\text { Focus on outages-slow } \\
\text { response to power quality } \\
\text { issues }\end{array}$ & $\begin{array}{c}\text { Power quality a priority with } \\
\text { a variety of quality/ price } \\
\text { options-rapid resolution of } \\
\text { issues }\end{array}$ \\
\hline $\begin{array}{l}\text { Optimization of assets and } \\
\text { operates efficiently }\end{array}$ & $\begin{array}{c}\text { Little integration of } \\
\text { operational data with asset } \\
\text { management-business process } \\
\text { silos }\end{array}$ & $\begin{array}{l}\text { Greatly expanded data } \\
\text { acquisition of grid parameters } \\
\text { focus on prevention, } \\
\text { minimizing impact to } \\
\text { consumers }\end{array}$ \\
\hline $\begin{array}{l}\text { Anticipating responses to } \\
\text { system disturbances } \\
\text { (self-healing) }\end{array}$ & $\begin{array}{l}\text { Responds to prevent further } \\
\text { damage; Focus on protecting } \\
\text { assets following a fault }\end{array}$ & $\begin{array}{l}\text { Automatically detects and } \\
\text { responds to problems; focus } \\
\text { on prevention, minimizing } \\
\text { impact to consumers }\end{array}$ \\
\hline $\begin{array}{l}\text { Resiliency against cyber } \\
\text { attack and natural disaster }\end{array}$ & $\begin{array}{l}\text { Vulnerable to malicious acts } \\
\text { of terror and natural disasters; } \\
\text { Slow response }\end{array}$ & $\begin{array}{l}\text { Resilient to cyber attack and } \\
\text { natural disaster; Rapid } \\
\text { restoration capabilities }\end{array}$ \\
\hline
\end{tabular}

The SG is expected to address the major shortcomings of the existing grid. In essence, SG will involve serious renewable energy resources. Hence, automated management is required for power system to ensure effective and efficient. In order to address these requirements, ICT is used in power grid. One of significant renovations is installation of SMs. SMs can provide two-way communications in real time between customers and utilities, which makes demand-side management possible. Table 1 describes the detail comparison between today's grid and SG.

HOMENET performs multiple important functions such as energy management, multimedia sharing, lighting control and climate control in SG [2], which should be addressed with priority. However, current protocols in HOMENET is based on host-centric IP protocol [3], which inherits serious fundamental problems of IP such as mobility, security and content multicasting distribution. Whereby, mobility and security are the basic requirements for HOMENET [2]. In order to solve these issues, ICN, with its specific features, are proposed for home communication fabric. The basic idea of ICN is to subscribe data in a content-centric manner irrespective of its location. Hence, users can only focus on what they are interested in, regardless of the physical address of content. Furthermore, ICN has pecial merit in delivering efficiency and network traffic reduction since ICN supports in-network caching. The same named data may get stored at multiple different locations. If the user changes location and the content is retrieved by re-expressing interests to the network, the data availability is improved under conditions of device mobility because, user can get data from nearest node which stored the data instead from the 
publisher. Moreover, the security of data relies on the data itself instead of communication channels. These features strongly benefit the current HOMENET. Last but not least, HOMENET is greater flexibility to be in particular with ICN because it is administratively-independent and smaller-scope [4][5]. [6]and[2] propose to apply ICN for HOMENET, but also present several challenges towards home automation. As one of these challenges, power management should be paid attention even though much related work has been done in IP-HOMENET. However, to our best acknowledge, few work focuses on REM in ICN-HOMENET until now, which is worth to be followed.

In the state-of-the-art REM implementation, SM are being deployed to homes in United States, Canada and Europe. Moreover, TOU rated are employed by several utility companies to enable flexible billing. Furthermore, the renewable energy, generated by home owners, can be consumed by home owners or sold to utility companies. Now a few projects are in trial and for example, Ontario government's Feed-In Tariff (micro-FIT) program [7] allows home owners to sell the excess energy generated by solar photovoltaics (PV), waterpower, biomass, wind, etc. to utility companies by specific contracts. Moreover, many "annually zero energy houses" are established in Toronto supported by NOW House project [8]. It means that the energy amounts these houses consume from grid is in equal to the energy amounts what they sell to grid in each year.

Recently, ICN-based HOMENET and REM scheme have become an active topic and several research work has been presented. In [2], the authors present a case for ICN based HOMENET in deal with the fundamental problems of IP-based network. Then a comparison in terms of service, control, and data plane complexity and features between IETF's HOMENET proposal with ICN-based approach is provided. Finally, proof-of-concept based analysis highlights the usefulness of ICN for HOMENET. However, this paper doesn't refer to any REM scheme for ICN-based HOMENET. [6] proposes a secure group communication scheme and an efficient group key management protocol specifically in ICN-based home communication fabric because secure group-oriented subscribe-publish communication (i.e. data confidentiality is guaranteed in multicasting) is not addressed by ICN. Whereas the REM scheme is not concerned by this paper. In [9], the authors propose a WSN-based energy control algorithm among intelligent devices for REM systems and the evaluation shows it is useful for cost-efficiency. However, in this case, the EMU doesn't cooperate with status measurement sensors to enable intelligent control decisions for actuation (e.g. close all the lights when none is detected). Furthermore, the "conflicting request" prevention mechanism is not referred and it is based on IP-HOMENET system. In summary, following ICN features, ICN-HOMENET is worth to keep researching specifically for REM scheme.

In our previous work, we proposed a modified REM algorithm to deal with conflicting requests of appliances [10]. In the regard, a domestic EMU is used to communicate with, manage and schedule all the appliances in HAN for saving bills. After received the appliance requests from users, EMU will firstly check the available self-generated energy (i.e. by solar panels, wind plants, etc.). If the local energy is not enough, EMU will communicate with SM to update the latest electricity price and peak hours information (i.e. TOU rates). Then it schedules the appliances' demands from peak hours to off-peak hours if possible to decrease power load and customer bills. Because the peaker plants are usually composed by coal and gas-fired plants during peak hours so that they have high maintenance and operation costs. Furthermore, this algorithm is experienced in a secure and mature cloud environment to test its large data processing capacity. Finally, a simulation-based evaluation demonstrates our proposed algorithm is available for TOU-aware HEM system and it is efficient to reduce consumers' bills and prevent network load increasing. However, all the communication technologies in this paper are based on IP protocol, which inherits serious fundamental issues of IP such as multicasting, security and mobility. In this paper, we presents a cost-efficient REM scheme for ICN based HOMENET in 
SG. First, in order to address the shortcomings of IP-HOEMENT such as security, mobility and content multicast distribution, we put forward to apply ICN in HOMENET system and because, it can provide natural support for above mentioned issues with the specific features of ICN such as self-contained data security, in-network caching and receiver-oriented operation. Meanwhile, ICN can support a much more efficient multimedia sharing in HOMENET due to its caching mechanism. Then, we present a cost-efficient REM scheme for ICN-HOMENET since it is very difficult to migrate existing IP-based REM schemes to ICN-based HOMENET, because they are two kinds of totally different Internet architectures. To the best of our knowledge, this is the first attempt to propose an ICN-based REM scheme in HOMENET system. In this proposal, we not only consider the conflicting requests from appliances and domestic power generation, but also think the EMU should cooperate with measurement sensors to control some specific appliances in specific conditions (e.g. EMU can send a request message to close the lights when nobody is measured at home). It is important to note that we should avoid this approach to decrease the comfort of the consumers.

The contributions of this paper are as follows:

1) We design an ICN approach specifically for HOMENET system called ICN-HOMENET to control network congestion, support mobility and ensure security. Moreover we conduct the proof-of-concept evaluation comparison between ICN-HOMENET system and existing HOMENET system based TCP/IP protocol.

2) We propose a cost-efficient REM scheme called ICN-REM scheme for ICN-HOMENET system which encourages consumers to shift the start time of appliances from peak hours to off-peak hours to reduce the energy bills. The corresponding performance evaluation validates its correctness and effectiveness. Moreover, a detailed comparison between the proposed ICN-REM scheme and other IP-REM schemes has been done.

The rest of the paper is organized as follows. In Section2, we introduce ICN approach that is available for the HOMENET. Section 3 presents a cost-efficient REM Scheme for ICN-HOMNET system in detail. And in Section 4 and 5, we give our proof-of-concept evaluation for ICN-HOMENET system and performance analysis of ICN-REM scheme, respectively. Finally, Section 6 concludes this paper.

\section{ICN-HOMENET SYSTEM}

\subsection{ICN-HOMENET Design Motivation}

HOMENET should support the multiple services such as light control, multimedia sharing, climate control and energy management [2]. Thus, it has strong requirements for security, mobility, network traffic control, etc. These problems also belong to the fundamental issues for IP network which need to be addressed with priority. However, ICN can help HOMENET in SG because ICN's features meet the HOMENET's requirements very well.

To begin with, ICN enforces receiver-oriented operation. It can help HOMENET provide the content-centric access manner. Normally, the users in HOMENET focus on the content itself what they are interested, rather than the content location. For instance, when a user tries to get one multimedia file in HOMENET, it is not necessary to get the physical address of this file. ICN can naturally address this requirement based on its "interest-data" paradigm through named-based routing. Moreover, shifting from host-centric to content-centric routing also makes it easier to support mobile clients. 


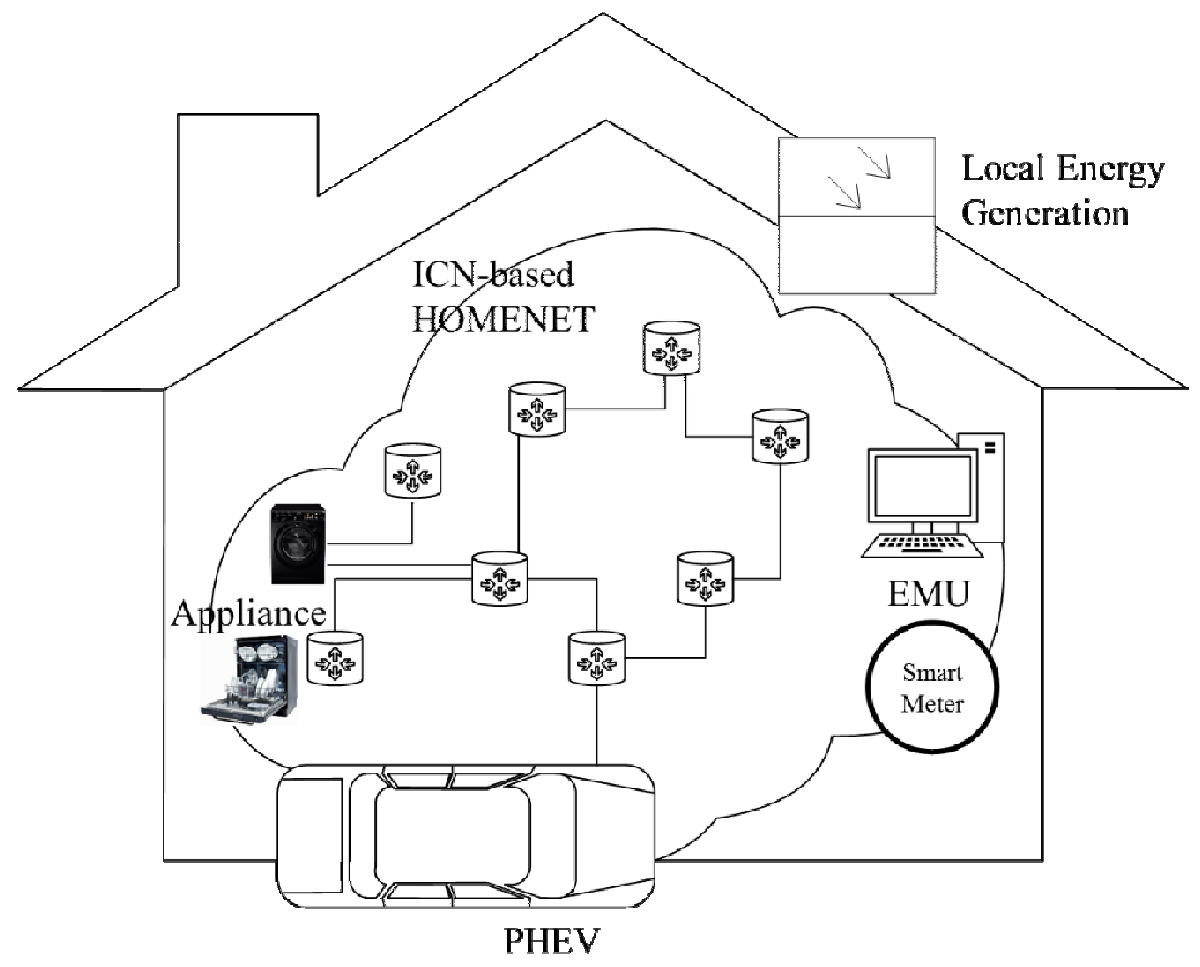

Figure 1. ICN-HOMENET System Structure [11]

Then, ICN supports in-network caching. It can efficiently reduce response time and transit traffic. Moreover, it is beneficial under mobility. Data can be cached at every intermediate node from a source to a destination in ICN, thus users can retrieve the data from the nearest router which stored the copy of data instead from the source again. This feature of ICN can help HOMENET provide better capabilities on network traffic control. In addition, the mobile devices in ICN don't need to retrieve data upon reconnection to the network but rather re-sending the "interest" and getting the data from the caching. Moreover, the temporarily disconnected devices also can benefit from in-network caching.

Last but not least, ICN secures the data itself instead of the protection of end-to-end communication channel. It can help HOMENET simplify security processing. HOMENET has a strong need to address security because large number of sensitive information such as customers' privacy in HOMENET should be protected. In comparison with IP network, ICN can provide stronger capabilities to ensure its confidentiality, integrity and authenticity. Besides, ICN's low-power operation and group-oriented "publisher-subscriber" manner are also beneficial to HOMENET.

\subsection{ICN-HOMENET System Structure}

Energy management unit, SM, local energy generation, EV and appliances serves as the crucial components to support the HOMENET. Now we propose a structure of ICN-HOMENET system (Figure 1) in term of existing literature [11]. The biggest difference is that the ICN-HOMENET replaces the IP routers in [11] to ICN routers. In addition, the structure is comprised of the following components: 
- SM comes with in-home displays, which measures and records the real-time energy usage and cost by two-way communication.

- Local energy generation is small scale electricity generation for family use and energy storage.

Table 2.Basic Notations and Definitions in ICN-HOMENET System

\begin{tabular}{cc}
\hline Notations & Meaning \\
\hline$i$ & The number of appliance \\
$R S t_{i}$ & The sequence number of request generated by the appliance, \\
storage and SM
\end{tabular}

- Energy management unit is responsible for communication with different other components to achieve the most efficient energy management.

- Appliance is short for intelligent devices which expects to shift its start time to off-peak hours to decrease energy bills.

- EV is powered through a collector system by electricity. And the electricity is provided by a battery or converted from fuel [12].

\subsection{Innovation from IP-HOMENET to ICN-HOMENET}

The migration from current IP-HOMENET to ICN-HOMENET can be gradual. One deployment option is based on an overlay model where ICN routers are selectively placed inside the current network. For example, ICN routers can be used as gateway nodes placed next to appliances for the purpose of efficient caching, aggregation, etc. This option is cost effective due to the use of IP networks without disturbing the underlying network infrastructure. The other option can be based on a clean-slate mechanism where the IP layer of the current network is totally replaced by the name or ID layer, and so name based routing is carried out in the pure ICN network.

\section{ICN-REM SCHEME FOR ICN-HOMENET SYSTEM}

\subsection{Basic Notations and Definitions for ICN-REM Scheme}

To design the ICN-REM scheme, the basic notations and definitions in ICN-HOMENET system is listed in Table 2. 


\subsection{Design Background and Principle for ICN-REM Scheme}

SMs and their communication with the grid, are the critical components of SG. With the installation of SMs, utilities are able to employ TOU pricing to enable flexible billing. According to the recently introduced TOU rates, price of electricity has been divided into peak, mid-peak and off-peak hours. Any appliance working in peak hours is required to charge more since the cost for generating power in peak hours is higher because, the peak plants must be brought online and cost more for maintenance and operation when customer demands exceed the capacity of

Table 3.Control Data of REM Scheme in ICN-HOMENET System

\begin{tabular}{ccc}
\hline Control Data & Name & "Data" content \\
\hline Start Request &.$/$ request/appliance/start & $i, s$, RSt $_{i}, t_{i}$ \\
Start Response &.$/$ response/appliance/start & $i, s, S S t_{i}$ \\
Start Notification &.$/$ /notification/appliance/start & $i, s$, ASt $t_{i}$ \\
Storage Request &.$/$ request/storage/available & $j, s$, AvailableStorageRequest \\
Storage Response &.$/$ response/storage/available & $j, s, E_{m}$ \\
Storage Update &.$/$ /update/storage/available & $j, s, i, F t_{i}, E_{m}$ \\
Meter Request &.$/$ request/meter/price & $s$, CurrentPriceRequest \\
Meter Response &.$/$ response/meter/price & $s, p_{t}$ \\
Stop Request &.$/$ request/appliance/stop & $i, s$, Reason \\
Stop Response &.$/$ response/appliance/stop & $i, s$, Reply \\
Control Request &.$/$ request/appliance/control & $i, s$, Reason \\
Control Response & ./response/appliance/control & $i, s$, Reply \\
\hline
\end{tabular}

base power plants in peak hours. However, this challenge can't be solved by easily updating the capacity of base power plants to match the peak load in peak hours due to the lack of large scale power storing technologies. Hence, it is a good choice to help customers decrease energy bills that shifting the power load from peak hours to off-peak hours. In REM Scheme, EMU, as a domestic management unit, communicates with appliances by Zigbee or WiFi technologies. Moreover, it also can exchange messages with the local storage center and SM to get the amount of available energy and update the price of electricity, respectively. In addition, sensors are widely used in smart home to guarantee security and measure health conditions. It is highly required to cooperate with EMU to provide much more efficient power management. Last but not the least, we also should highlight the conflicting request in REM because it will cause the network traffic even network paralysis.

Hence, the design principle of ICN-REM scheme is listed as follows.

1. ICN-REM scheme should extremely improve efficiency and decrease the bills of customers. In the regard, it is encouraged to shift demands to off-peak hours at the most extent.

2. EMU should cooperate with local generated energy from solar, wind and so on.

3. Conflicting requests from appliances should be extremely avoided.

4. ICN-REM should cooperate with home sensors to improve efficiency of energy management.

5. EMU can't force any automated start time on the appliances because this will cause discomfort on customers' side.

\subsection{Preparation for ICN-REM Scheme}


In ICN-HOMENET system, "subscribe-publish" paradigm can't address the requirements of monitor for publisher. For instance, in order to receive the start request message from appliances, the EMU must send the "interest" uninterruptedly. [6] has proposed APIs to solve this problem. It can send "interest" only one time to keep itself available once, periodically or persistently.

a) Once. The subscription only keeps available once after "interest" arrived publisher side.

b)Periodic. The subscription keeps available in publisher side periodically such as one hour, one day.

c)Persistent. The subscription is kept persistent.

\subsection{The Process of ICN-REM Scheme for ICN-HOMENET System}

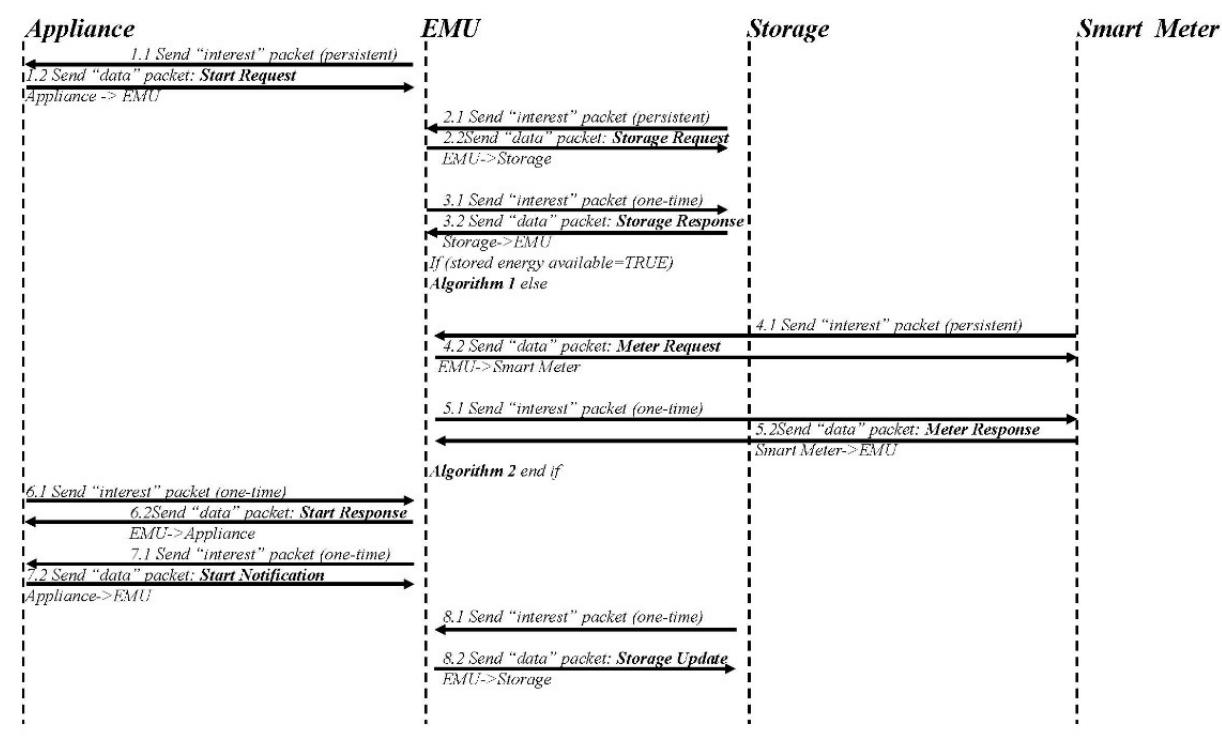

Figure 2. Flow Chat of ICN-REM Scheme (1)

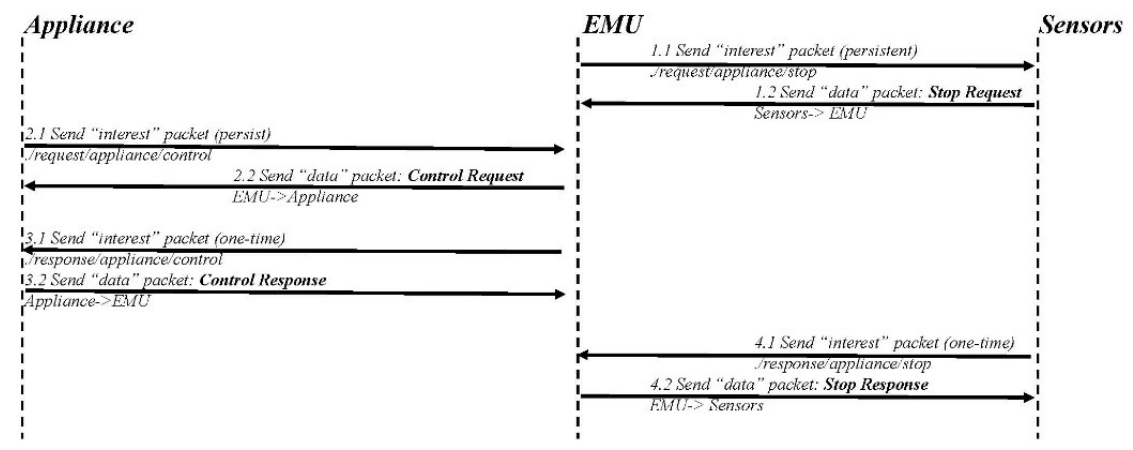

Figure 3. Flow Chat of ICN-REM Scheme (2)

For REM in ICN-HOMENET environment, the control data are illustrated in Table 3. Start Request/Start Response are used for an appliance to inform EMU that it plans to work and then get a suggested start time from EMU. Start Notification is used for appliance to inform EMU its 
actual start time, which is absolutely decided by consumer. The detailed reason is explained in section 3.2. Storage Request / Storage Response / Storage Update are used for EMU to send request of the amount of available storage to local power generation, get the response of the amount, and inform how much local power left after it is used by any appliance.

Meter Request/Meter Response are responsible for getting the price of electricity from SM. All above control data are used for Flow Chat of ICN-REM Scheme (1) (Figure. 2). Moreover, Stop Request/Stop Response and Control Request / Control Responseare used for EMU to cooperate with large number of home sensors to improve the efficiency of ICN-REM scheme. The detailed scenario is illustrated in Figure 3.

In ICN-REM scheme, we consider two different kinds of scenarios. One scenario is that EMU communicates with storage, SM and appliance to extremely shift the start time of demands to off-peak hours in purpose of saving customers' bills, which is called ICN-REM scheme (1) and the flow chat is proposed in Figure 2. The other scenario is that EMU cooperates with home sensors to provide more efficient REM scheme (i.e. EMU can suggest appliances to be closed or changed to sleep mode when it detect unattended appliances from sensor signals), which is called ICN-REM scheme (2) and flow chat is proposed in Figure 3.

Table 4. Algorithm 1 - Scheduling at the EMU when the Stored Energy is Available.

\begin{tabular}{l} 
Algorithm 1 - Scheduling at the EMU when the Stored Energy is Available. \\
\hline 1: if $\left(R S t_{i}\right.$ is a conflicting request $)$ then \\
2: $S S t_{i} \leftarrow$ ShiftToDelay( $)$ \\
3: else $^{\text {4: } S S t_{i} \leftarrow R S t_{i}}$ \\
5: end if
\end{tabular}

The detailed analysis for Figure 2 is highlighted to be shown as follows.

1) Step 1: Start Request will be sent from Appliance to EMU if it plans to work. Wherein, the requested start time $\left(R S t_{i}\right)$, operated time duration $\left(D t_{i}\right)$ and others will be involved in this message.

2) Step 2: After received the Start Request, EMU will communicate with Storage to check the amount of available local energy from Storage by message Storage Request.

3) Step 3: After received the available energy amount from Storage Response, the Algorithm 1 will calculate the suggested start time of appliance if the stored energy is enough for this appliance. Else

4) Step 4: EMU will send a request for getting the price of electricity and peak information from SM by Meter Request.

5) Step 5: After received the above information by Meter Response, it will run Algorithm 2 to calculate the suggested start time of appliance.

6) Step 6: Then the suggested start time will be sent back to appliance by Start Response.

7) Step 7: After appliance decided the actual start time, it will be sent to EMU by Start Notification. It is worth noting that all the actual start time should be decided by appliance itself and EMU can't force any automated start time on the appliances because this will cause discomfort on customers' side.

8) Step 8: Finally, the used amount of local energy will be reported to Storage by Storage Update. In this scheme, if appliance can absolutely consume the local energy, it only needs to consider the conflicting requests regardless of TOU rates because local energy is no charging for consumers. This is the reason why EMU will not communicate SM to calculate the suggested start time of appliance. The detailed Algorithm 1 (i.e. Scheduling at the EMU when stored energy is available) 
International Journal of Computer Networks \& Communications (IJCNC) Vol.8, No.2, March 2016

is shown in Table 4. Moreover, our previous work in [10] also contributes some details in following algorithm.

Then, the detailed Algorithm 2 (Scheduling at the EMU when the stored energy is not available) is illustrated in Table 5 as follows. In brief, if the requested start time of appliance isin peak hours, it will be encouraged to shift the start time to off-peak hours or mid-peak hours. Or if the requested start time of demand is in mid-peak hours, it will be encouraged to shift to off-peak hours. During these processes, the maximum allowable delay and conflicting request are considered. Moreover, our previous contribution [10] has some details, which will not list in this paper.

Since EMU cooperates with home sensors, it should put forward a novel ICN-REM scheme. In this scheme, four kinds of control messages such as Stop Request, Stop Response, Control Request and Control Response are used to assist EMU for this energy-efficient function in ICN-REM.

Table 5.Algorithm 2 - Scheduling at the EMU when the Stored Energy is Not Available

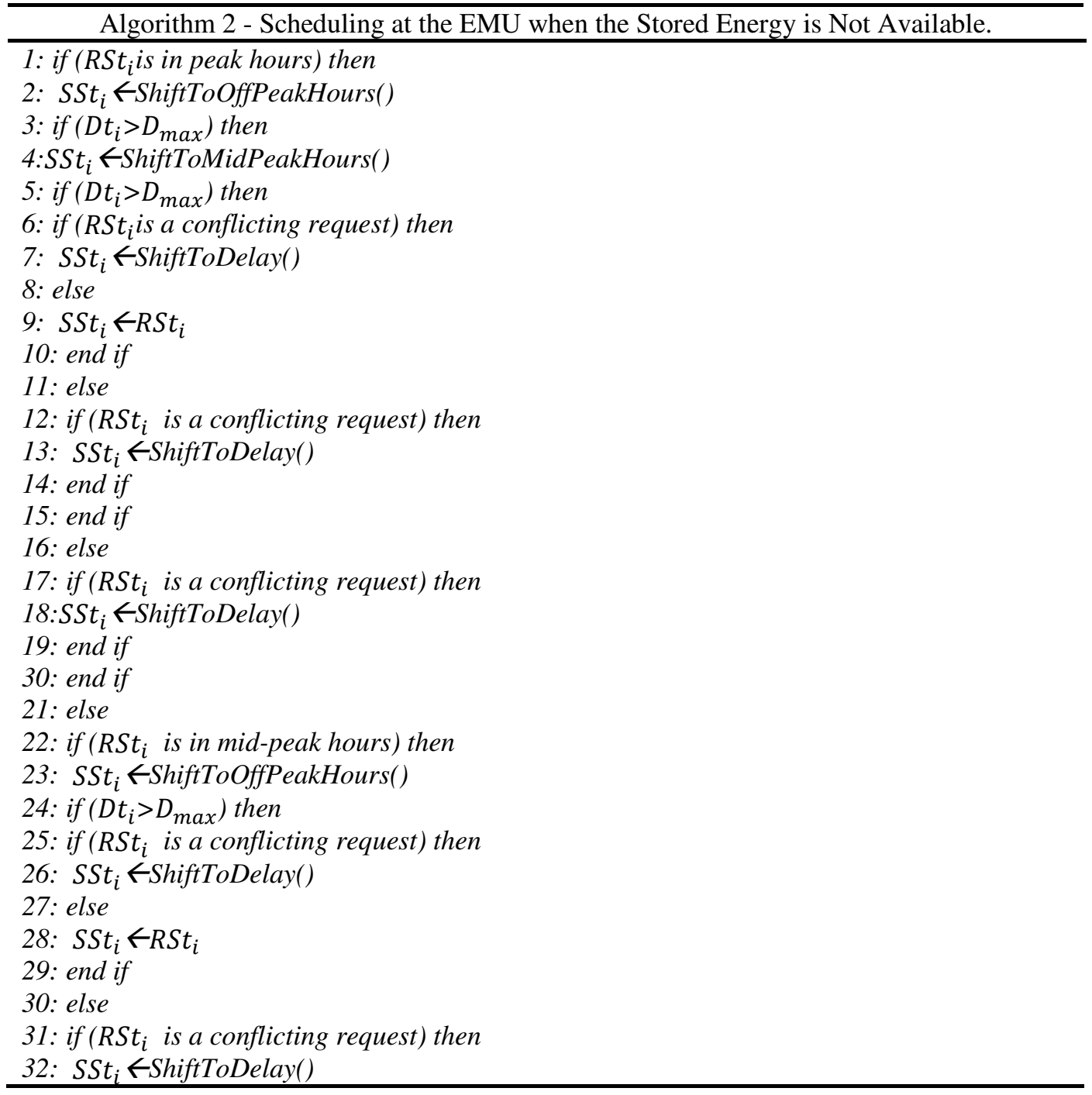




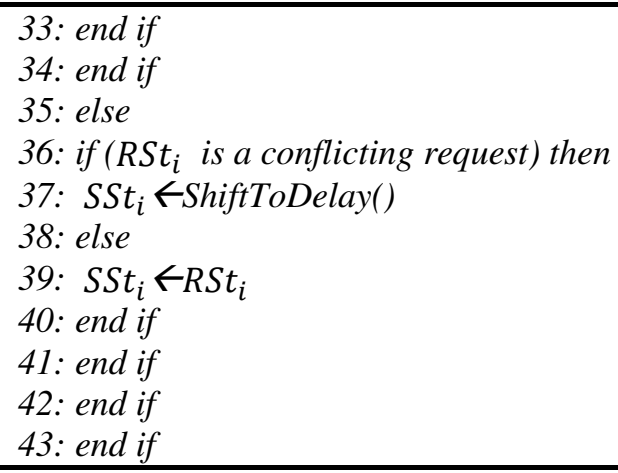

Hereby, the detailed 4 steps for ICN-REM Scheme (2) (Figure 3) is highlighted to be shown as follows.

1) Step 1: Sensors will send message Stop Request to EMU if Sensors measure any appliance is unattended. (e.g. lights are working but nobody is in this room).

2) Step 2: Then EMU will inform the corresponding appliance by a message Control Request with some control request. For instance, request for closing the appliance, changing to sleep mode or others.

3) Step 3: If the customer agrees this request by local or remote operation (i.e. smart phone or other devices), it will notice EMU by Control Response. It is worth noting that all the actual start time should be decided by appliance itself and EMU can't force any automated start time on the appliances because this will cause discomfort on customers' side.

4) Step 4: Finally, EMU will send a message Stop Response to corresponding sensors' request before.

\section{Proof-of-Concept Evaluation for ICN-HOMENET}

In this paper, we will not give a numeric evaluation between ICN-HOMENET and IP-HOMENET because, the emphasis of this paper is ICN-REM scheme rather than performance evaluation of ICN-HOMENET. Furthermore, [2] and [6] already provided enough results that demonstrate ICN approach can well support for HOMENET system. Moreover, we will present a proof-of-concept evaluation to deal with the HOMENET issues in IP environment illustrated in this paper as follows.

Security. ICN's self-contained data security can help HOMENET system simplify security processing. In HOMENET system, it has a strong requirement to address security, especially confidentiality (requirement that data is intelligible only to authorized entities), integrity (requirement that data is the same as the source), and authenticity (requirement that data is from who it says it is from). Because energy data in HOMENET system can reflect our living privacy such as someone is at home or not, eating habits, health conditions, etc. However, the security of IP-HOMENET system is based on protection of end-to-end communication channels, which is an inheriting shortcoming from IP protocol and easy to be attacked by adversary. In the contrast, ICN-HOMENET system relies on the protection of data itself to ensure data integrity as well as authenticity by signature and confidentiality by encryption at data creation.

Mobility. ICN approach can help HOMENET system support mobility when devices change the network at home. HOMENET has a strong mobility requirement to ensure the seamless multimedia sharing when user switches the network. From the concept, ICN focuses on the content customers want to access regardless of where (on what host) that content resides, which helps ICN provide the foundation of mobility support. Moreover, on teh basis of name-based 
routing and distributed data caching in ICN approach. The mobile devices in ICN do not need to retrieve data upon reconnection to the network instead of re-sending the "interest" and getting the data from the caching of nearest router which has stored the copy of data. It fully satisfies the need of HOEMNET system, since devices in AMI may be only intermittently connected due to mobility.

Multicasting. ICN approach can help HOMENET system provide group-oriented publish-subscriber (pub-sub) manner. The group-oriented pub-sub communication manner is largely used among devices in HOEMNET systems. For example, the EV charging information will be sent to multiple places such as EV dashboard, owners' laptop, phone, etc. Actually, ICN's "interest-data" model maps naturally to data sharing pub-sub groups, and the entries in FIB and PIT tables map to a group of devices interested in the same piece of data.

In summary, ICN well suits the requirements of HOMENET system from security, mobility and group-oriented communication support. It can provide better services for future HOMENET system.

\section{Performance Analysis of ICN-REM Scheme}

Table 6.Power rate and duration of appliances

\begin{tabular}{lcccc}
\hline & Washer & Dryer & Dish-washer & Coffee maker \\
\hline Power Rate $(\mathrm{kW} / \mathrm{h})$ & 0.89 & 2.46 & 1.19 & 0.4 \\
\hline Working Duration $($ min) & 30 & 60 & 90 & 10 \\
\hline
\end{tabular}

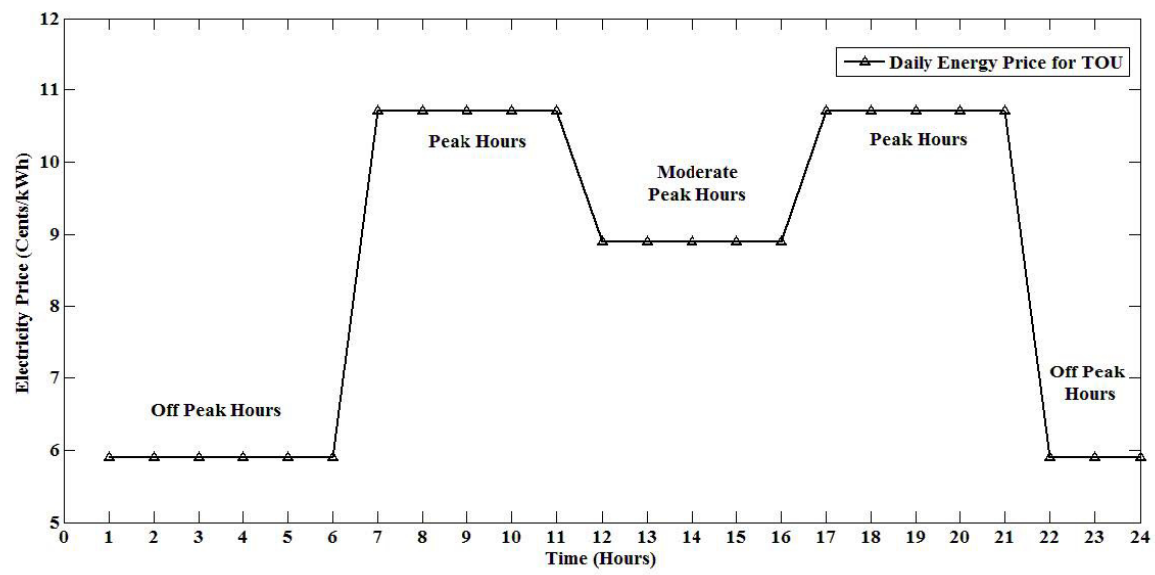

Figure 4.Daily Energy Price for TOU

We use NDNSim[13] for our simulations and part of simulation metrics and parameters follow [9][14][11]. There are four different home appliances are used in this simulation, which are washer, dryer, dishwasher and coffee maker. Furthermore, the power rate and working duration of each appliances (Shown as in Table 6) can be found in [15]. Specifically the power rates of washer, dryer, dishwasher and coffee maker are $0.89 \mathrm{~kW} / \mathrm{h}, 2.46 \mathrm{~kW} / \mathrm{h}, 1.19 \mathrm{~kW} / \mathrm{h}$ and $0.4 \mathrm{~kW} / \mathrm{h}$, respectively. And the working durations of each appliances are 30 minutes, 60 minutes, 90 
minutes and 10 minutes. Moreover, the coffee maker is assumed to be used for making 2 cups of coffee.

In our simulation, refer to the Hydro Ottawa daily energy price of TOU in winter condition [16], it is illustrated in Figure 4. Wherein, one day has been divided into three different durations: peak hours from 7 am to $11 \mathrm{am}$ and from $5 \mathrm{pm}$ to $9 \mathrm{pm}$, mid-peak hours from $11 \mathrm{am}$ to $5 \mathrm{pm}$, and the rest of time in one day is off-peak hours. Meanwhile, the respective energy price is 10.7 cents/kWh in peak hours, 8.9 cents $/ \mathrm{kWh}$ in moderate hours, and 5.9 cents $/ \mathrm{kWh}$ in off peak hours. Furthermore, consumer demand is modelled as a Poisson process to address the increasing demands in peak hours. And the solar power assumed to be generated with 6 PV panels with each having a capacity of $350 \mathrm{Wh}$ per day. This amount is approximately equal with the actual power generation by one solar panel with two hours of effective energy generation in winter.

In Figure 5, it compares the total cost of one home within 100 days in four different cases. It testifies the efficiency of ICN-REM scheme for ICN-HOMENET system and it also shows the significance of local energy generation for customers' bills. Note that total cost increase with increasing days because the bill is calculated cumulatively. As seen in Figure 5, the cost of customer with ICN-REM and local energy is more than 5 times than customers without them. Moreover, it demonstrates that shifting the appliance to off-peak hours is an efficient way to decrease bills for TOU-aware energy system.

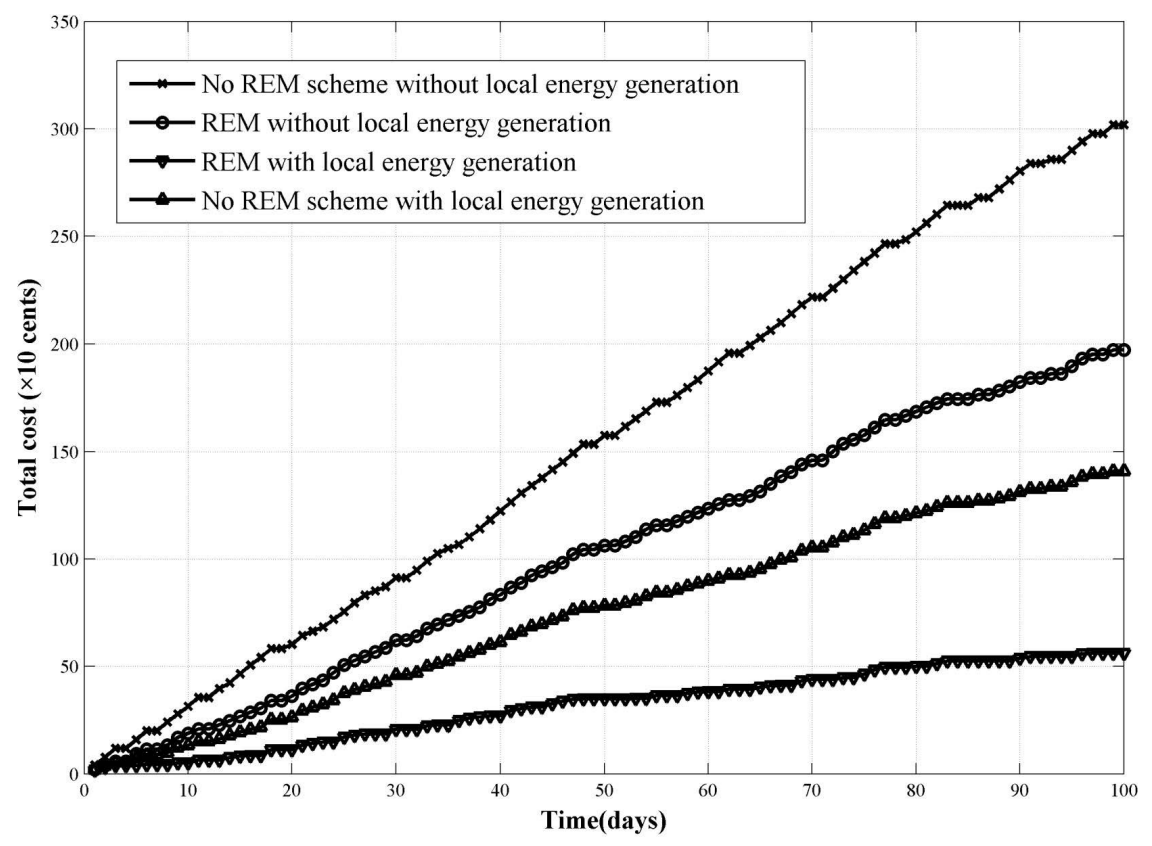

Figure 5.The Total Cost Comparison 


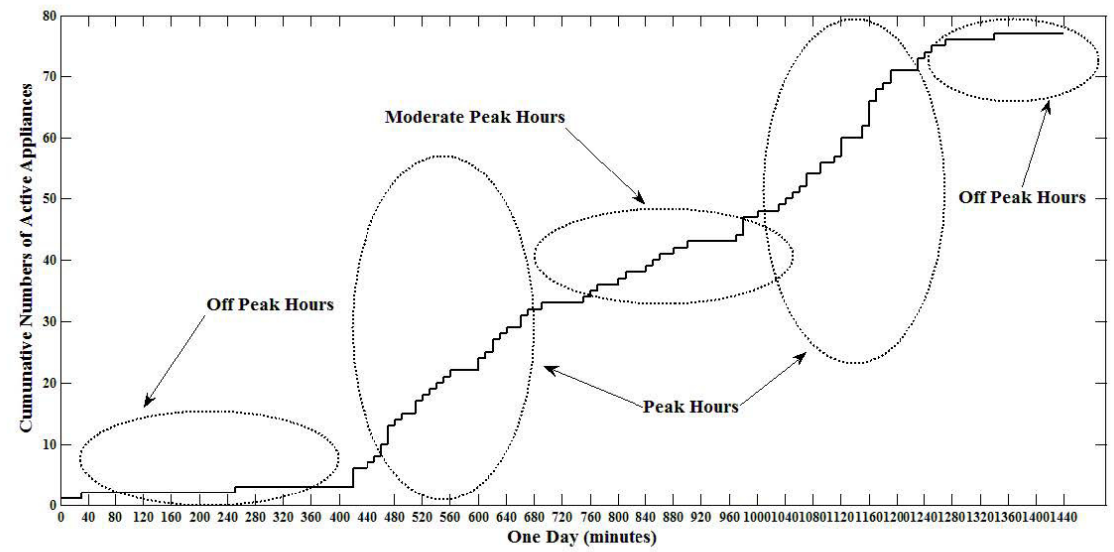

Figure 6.Cumulative Numbers of Active appliances in the Nature Condition (No REM and CRA)

In ICN-REM scheme, Conflicting Request Avoiding (CRA) is one of ICN-REM design principles and requirements, which is often ignored in ICN-REM design. Large number of conflicting request may cause network congestion. In order to demonstrate our proposed ICN-REM is efficient for CRA, we use cumulative distribution function to show the cumulative numbers of active appliances in Figure 6,7 and 8. The horizontal axis stands for one day, here it is shown by minutes rather than hours. Moreover, the vertical axis means the cumulative numbers of active

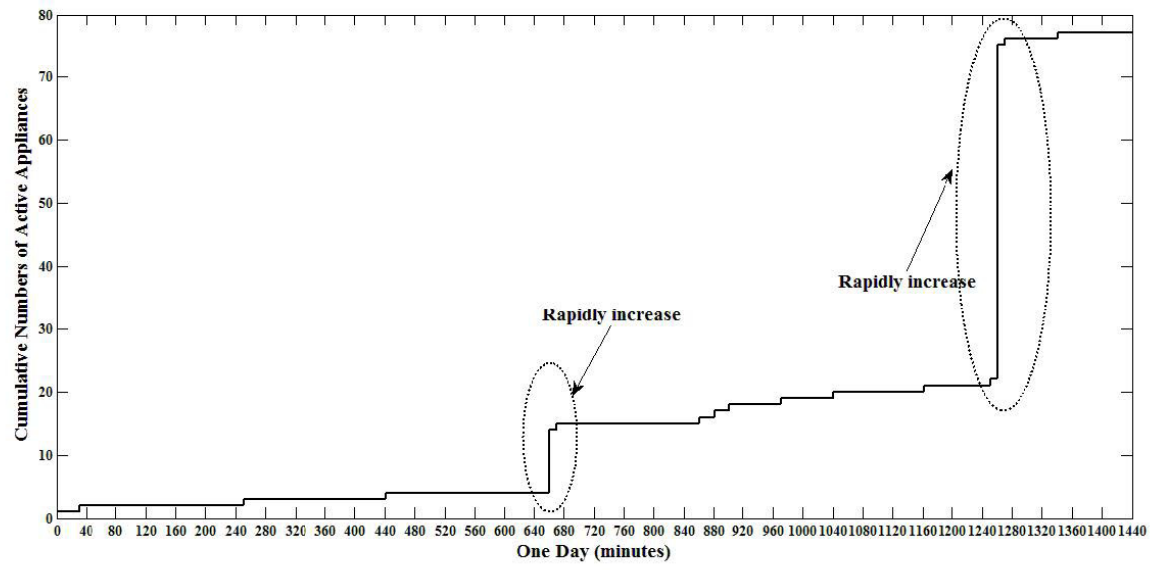

Figure 7.Cumulative Numbers of Active Appliances based on ICN-REM (No CRA) 


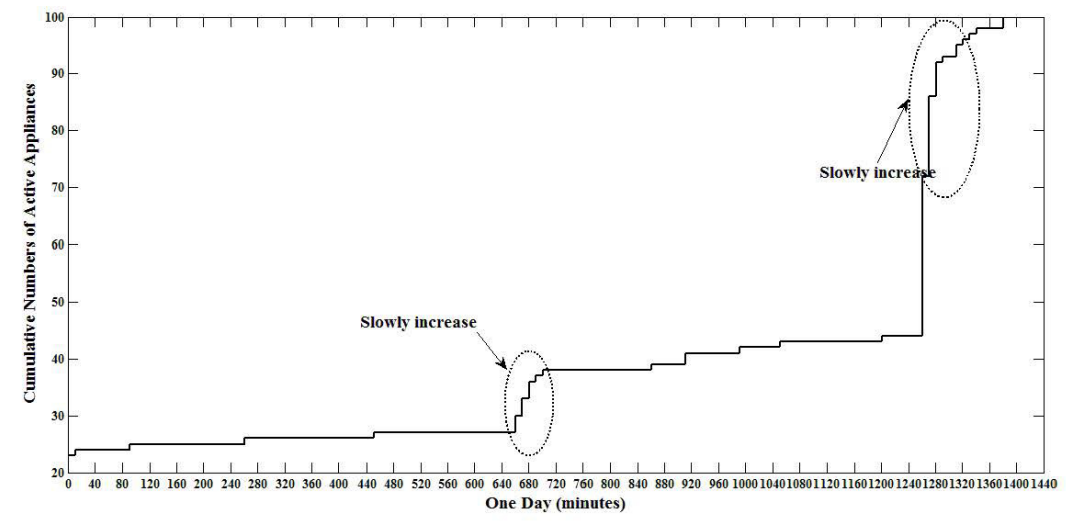

Figure 8.Cumulative Numbers of Active Appliances based on ICN-REM (CRA)

appliances. In these figures, if the cumulative numbers of active appliances rapidly increase, it shows large number of conflicting requests are existing.

Figure 6 illustrates the cumulative numbers of active appliances in the nature condition (i.e. there is no REM scheme and CRA mechanism). In this figure, the number of active appliances rapidly increases in two time intervals, which is respectively from 421 minutes (7:00 am) to 660 minutes $(11: 00 \mathrm{am})$ and from 1021 minutes (17:01) to 1260 minutes (21:00). It is approximately same with the peak hours' period. It also shows the distribution of demands almost concentrates in the peak or moderate peak hours the nature condition.

In the Figure 7, it illustrates the cumulative numbers of active appliances based on ICN-REM scheme but the CRA mechanism is not used in this case [9]. It shows the period of rapid increasing moves toward right direction in $\mathrm{x}$ axis. Particularly shift from 421 minutes (07:01 am) to 661 minutes $(11: 01 \mathrm{am})$ and from 1021 minutes $(17: 01)$ to 1261 minutes $(21: 01)$, it means the demands of appliances can efficiently shift from peak hours to moderate peak hours or off peak hours based on REM Scheme. On the other hand, the rapid increase also states the shifted demands largely aggregate in some same timeslots in off peak hours. In other words, large number of conflicting requests work. It may increase the network load and cause new peak hours.

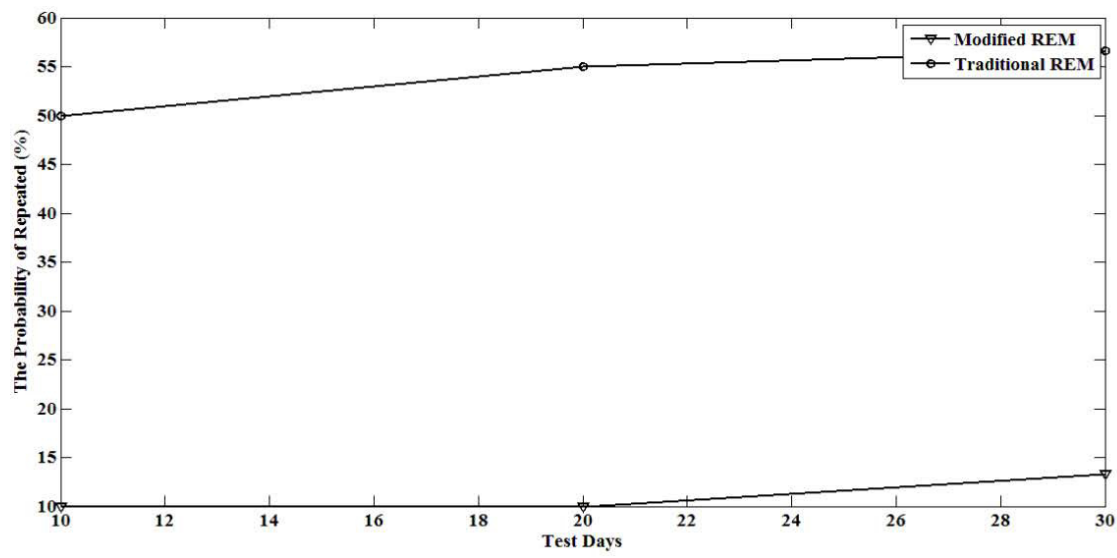

Figure 9.Conflict probability of demand between ICN-REM and [9] 
In order to better evaluate this our proposed ICN-REM scheme, the numerical verification is used in Figure 8. Compared with Figure 7, the period of rapid increasing can also successfully move toward right direction in $\mathrm{x}$ axis. In other word, it also can effectively change the demand from peak or moderate peak hours to off peak hours. But the cumulative numbers of active appliances can be much slower increase in off peak hours. It clearly shows that demand can be effectively shift to the off peak hours and it also can prevent conflicting requests.

From the Figure 9, it more clearly demonstrates that our proposed ICN-REM scheme can efficiently decrease the conflict probability of demand. By our proposed scheme, the probability of conflict can decrease from $50 \%$ to $10 \%$ in test 10 days. Moreover if the experience period extends to 30 days, conflict probability can be decreased from 55\% to 15\%. This also demonstrates the proposed scheme is excellent to prevent the conflicting requests.

\section{CONCLuSiON}

HOMENET plays an important role in SG since it performs multiple functions such as energy management, multimedia sharing, climate and lighting control, etc. However, current IP-HOMENET inherits serous fundamental problems of IP protocol such as mobility, group-oriented manner and mobility. In order to solve these issues, we put forward to apply ICN in HOMENET system because it can provide natural support for security, mobility and multicasting. Then we conduct the proof-of-concept evaluation comparison between ICN-HOMENET system and IP-HOMENET system. Furthermore, we present a cost-efficient REM scheme called ICN-REM for ICN-HOMENET system which encourage consumers to shift the appliance from peak hours to off-peak hours to reduce the energy bills. In this proposal, we not only consider the conflicting requests from appliances and domestic power generation, but also think the EMU should cooperate with measurement sensors to control some specific appliances in specific conditions. The corresponding performance evaluation validates its correctness and effectiveness. To the best of our knowledge, this is the first attempt to propose an ICN-based REM scheme in HOMENET system.

\section{REFERENCES}

[1] Kevin P Schneider, Yousu Chen, David P Chassin, Robert G Pratt, David W Engel, and Sandra Thompson. Modern grid initiative: Distribution taxonomy final report. Pacific Northwest National Laboratory, 2008.

[2] R. Ravindran, T. Biswas, Xinwen Zhang, A. Chakraborti, and Guoqiang Wang. Information-centric networking based homenet. In Integrated Network Management (IM 2013), 2013 IFIP/IEEE International Symposium on, pages 1102-1108, May 2013.

[3] JariArkko, Jason Weil, Ole Troan, and Anders Brandt. Home networking architecture for ipv6. 2012.

[4] Van Jacobson, Diana K. Smetters, James D. Thornton, Michael Plass, Nick Briggs, and Rebecca Braynard. Networking named content. Commun. ACM, 55(1):117-124, January 2012.

[5] Dirk Trossen, MikkoSarela, and Karen Sollins. Arguments for an information-centric internetworking architecture. ACM SIGCOMM Computer Communication Review, 40(2):26-33, 2010.

[6] Jianqing Zhang, Qinghua Li, and E.M. Schooler. ihems: An information-centric approach to secure home energy management. In Smart Grid Communications (SmartGridComm), 2012 IEEE Third International Conference on, pages 217-222, Nov 2012.

[7] Ontario Power Authority, Feed-In-Tari_program. http://fit.powerauthority.on.ca.

[8] The Now House Project. http://www.nowhouseproject.com.

[9] MelikeErol-Kantarci and Hussein T Mouftah. Wireless sensor networks for cost-efficient residential energy management in the smart grid. Smart Grid, IEEE Transactions on, 2(2):314-325, 2011.

[10] Keping Yu, Zhenyu Zhou, and Takuro Sato. Cloud-based modified residential energy management algorithm in smart grid network. In InternationalConference on Modeling and Simulation Technology (JSST 2013), Sep 2013. 
[11] MelikeErol-Kantarci and Hussein $\mathrm{T}$ Mouftah. Tou-aware energy management and wireless sensor networks for reducing peak load in smart grids. In Vehicular Technology Conference Fall (VTC 2010-Fall), 2010 IEEE 72nd, pages 1-5. IEEE, 2010.

[12] Keping Yu, Zhenyu Zhou, and Takuro Sato. Performance evaluation of residential energy management algorithm in smart grid network. In 2011 IEICE Society Conference, September 2011.

[13] Alexander Afanasyev, IlyaMoiseenko, Lixia Zhang, et al. ndnsim: Ndn simulator for ns-3. University of California, Los Angeles, Tech. Rep, 2012.

[14] MelikeErol-Kantarci and Hussein $T$ Mouftah. Wireless sensor networks for domestic energy management in smart grids. In Communications (QBSC), 2010 25th Biennial Symposium on, pages 63-66. IEEE, 2010.

[15] R. Stamminge. Synergy potential of smart appliances, deliverable 2.3 of work package 2 from the smart-project. University of Bonn, March 2009.

[16] Hydro Ottawa TOU rates. http://www.hydroottawa.com.

\section{AUTHORS}

Keping Yu was born in China, on January 1988. He received his B.E. and B.Admin. degree from Sichuan Normal University, Sichuan, China in 2010 and University of Electronic Science and Technology of China, Sichuan, China in 2010, respectively. He received his M.Sc. degree in Wireless Communication from Waseda University, Tokyo, Japan in 2012. Currently, he is a Ph.D. candidate at Graduate School of Global Information and Telecommunication Studies (GITS), Waseda University, Tokyo, Japan. He is a student member of IEEE. His research interests include smart grid, content-centric networking and their information security.

BattulgaDavaasambuu received the BS and MS degrees in computer engineering from National University of Mongolia, Mongolia, in 2007 and 2009, respectively. From 2009 to 2011, he worked as a research engineer at the National University of Mongolia. He is currently a Ph.D. candidate in the Graduate School of Global Information and Telecommunication Studies, Waseda University, Tokyo, Japan. His current research interests include ICT, mobility management, and wireless networking.

Nam Nguyen received the Master Degree in Information and Communication from Graduate School of Global Information and Telecommunication Studies (GITS), Waseda University, Japan in 2012. Currently, he is a PhD candidate at GITS, Waseda University. His research interests are in the area of HetNet, Wi-Fi offloading for Cellular Network. He received Japan Government Scholarship since 2010.

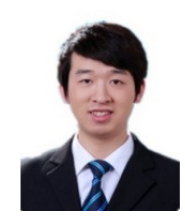

Quang Ngoc NGUYEN was born in Ha Noi, Vietnam. He received the B.E degree in Information Technology,Honor Computer Science Program conducted in English from Posts and Telecommunications Institute of Technology (PTIT), Ha Noi, Vietnam in 2012. After that, he was asked to stay to work at PTIT and became one of the youngest members of Institute. He was involved in building documents for opening new major in Information Security, which is the first and pilot Regular Undergraduate program in Information

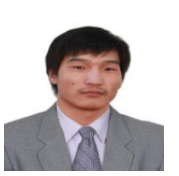
Security in Vietnamese education. He was the sole Awardee of Asia Special Scholarship, Waseda University for Fall 2013 admission to Graduate School of Global Information and Telecommunication Studies (GITS), Waseda University, Tokyo, Japan. Currently, he is pursuing M.S degree in Computer Systems and Network Engineering Area at GITS. His research interests include Future Internet Architecture, Green Network, Information Centric Networking and Next Generation Mobile Communication Systems. 
Mohammad Arifuzzaman received the B.Sc. degree in Computer Science \& Engineering from Bangladesh University of Engineering and Technology (BUET) in 2001. He worked as an Assistant professor at IBAIS University, Dhaka, Bangladesh from 2001 to 2005. After that he joined in the Bangladesh Civil Service in 2006 and worked as an Assistant secretary to the Government of the People's Republic of Bangladesh till 2010. He has completed

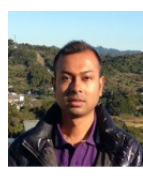
Masters in Global Information and Telecommunication StudiesfromWaseda University, Tokyo, Japan in 2012. Now he is a PhD candidate at GITS ofWaseda University. He received many awards including the best paper award in the ITU Kaleidoscope Conference, Cape Town, South Africa, 12-14 December 2011. His research interests lie in the area of Communication protocols, wireless ad-hoc and sensor networks, Next Generation Mobile communication systems and Future Internet Architecture. He is a student member of IEEE.

Takuro Sato received the B.E. and Ph.D. degrees in Electronics Engineering from Niigata University in 1973 and 1993 respectively. He joined the Research and Development Laboratories of OKI Electric Industry Co., Ltd., Tokyo, Japan in 1973 and he has been engaged in research on PCM transmission equipment, mobile communications, data transmission technology and digital signal processing technology. He developed wideband CDMA system for personal communications system and joined the PCS standardization

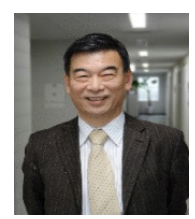
committee in USA and Japan. He contributed in high speed cellular modem standardization for ITU, 2.4GHz PCS standardization for ITA and wireless LAN standardization for IEEE 802.11. He was a Senior Research Manager and Research Director in Communication Systems Laboratory of OKI Electric Industry Co., Ltd. He served as a professor of Niigata Institute of Technology from 1995 and he researched on CDMA, OFDM, personal communication systems and related area. In 2004, he joined as a professor of GITS atWaseda University and currently serving as a Dean of the Graduate School of Global Information and Telecommunication Studies (GITS), Waseda University. His current research interests include Wireless Sensor Network, Mobile IP Network, ICT in Smart Grid, 4G mobile communication systems. He is Fellow of IEICE and IEEE. 